\title{
Registro e perfil ecotoxicológico de produtos para controle de Aedes aegypti
}

\section{Registry and ecotoxicological data of Aedes aegypti control products}

\author{
Natália Mendes Gomes \\ Magalhães ${ }^{1, *}$ iD \\ Raquel Lima e Silva" \\ Laila Salmen Espindola'
}

' Laboratório da Farmacognosia, Universidade de Brasília (UnB), Brasília, DF, Brasil

" Agência Nacional de Vigilância Sanitária, Brasília, DF, Brasil

\footnotetext{
* E-mail: nataliamendesgomes@gmail. com
}

Recebido: 20 jan 2020

Aprovado: 05 nov 2020

\begin{abstract}
RESUMO
Introdução: Arboviroses transmitidas pelo Aedes aegypti têm grande relevância para a saúde pública, sendo o controle químico do vetor uma importante estratégia de mitigação. Entretanto, o uso intensivo de pesticidas está associado a seleção de insetos resistentes e impacto ambiental. Por isso, é essencial desenvolver e regulamentar novos produtos, eficazes e sustentáveis. Objetivo: Descrever os requisitos para regulação de inseticidas e repelentes no Brasil, à luz de orientações internacionais. Comparar os ativos regulamentados no país com os disponíveis nos Estados Unidos (EUA) e União Europeia (UE). Por fim, realizar um levantamento de estudos ecotoxicológicos dos ativos empregados no controle vetorial. Método: Revisão narrativa de instrumentos regulatórios nacionais e internacionais, literatura científica, consulta a agências regulatórias e ao ECOTOX Knowledgebase. Resultados: As normas brasileiras trazem exigências consoantes com as internacionais. Todos os ativos disponíveis nos EUA têm monografia aprovada pela Anvisa; já a UE mostrou-se mais restritiva. Há menos repelentes tópicos disponíveis no Brasil e na UE, comparando-se com EUA. Muitos dos inseticidas aprovados para controle vetorial representam risco para organismos não alvo. Ainda assim, não identificamos quaisquer estudos para alguns dos produtos no banco de dados pesquisado. Conclusões: A UE mostrou-se a agência mais restritiva com relação ao registro de inseticidas. Existe a necessidade de guias com orientações específicas sobre o desenvolvimento de produtos inovadores para o controle do mosquito, bem como de ferramentas de busca com maior transparência na página da agência brasileira. Finalmente, esta revisão reitera a importância de análises ecotoxicológicas mais abrangentes para os produtos em questão, considerando seu potencial dano ambiental.
\end{abstract}

PALAVRAS-CHAVE: Arboviroses; Aedes aegypti; Registro; Inseticidas; Toxicidade

\section{ABSTRACT}

Introduction: Arboviruses transmitted by Aedes aegypti have big relevance for public health worldwide, with chemical vector control being an important mitigation strategy. Nevertheless, the intensive use of insecticides is associated with resistance and environmental toxicity. That is why it is essential to develop and regulate new products, effective and sustainable. Objective: To describe the requirements for regulation of insecticides and repellents in Brazil, in the light of international guidelines. To present, comparatively, the active ingredients approved in Brazil, United States (USA) and the European Union (EU). Finally, to conduct a survey of ecotoxicological studies from active ingredients used for vector control. Method: Narrative review of national and international regulatory instruments, scientific literature, consultation with regulatory agencies and assess to ECOTOX Knowledgebase. Results: Brazilian requirements are in line with international standards. All insecticides active ingredients available in the USA have a monograph approved in Brazil by the National Health Surveillance Agency; the EU was more restrictive. There are fewer topical repellents available in Brazil and in the EU, compared to the USA. Many of the insecticides approved for vector control pose a risk to 
non-target organisms. However, we did not identify studies for some of them in the researched database. Conclusions: The EU seems to be the most restrictive agency when it comes to registering insecticides. Specific guides on development of innovative products for vector control are needed, as well as greater transparency in research instruments at the Brazilian agency website. This review also reiterates the necessity of more ecotoxicological analysis regarding insecticides, considering their potential environmental hazard.

KEYWORDS: Arboviruses; Aedes aegypti; Registry; Insecticides; Toxicity

\section{INTRODUÇÃOO}

Arboviroses, como dengue, zika, chikungunya e febre amarela, são doenças infecciosas tropicais significativamente impactantes para a saúde pública e possuem como principal vetor o mosquito Aedes aegypti1,2. Nas últimas duas décadas, dengue e chikungunya tornaram-se as arboviroses de maior impacto mundial, devido ao aumento de casos de transmissão. No Brasil, dados de 2016 revelaram que a dengue foi a doença de maior relevância sanitária no período, tendo afetado cerca de 1,5 milhão de pessoas entre 2015 e 2016 ${ }^{3}$. Em 2019, até o mês de agosto, foram registrados mais de 1,4 milhão de casos da doença ${ }^{4}$.

Os tratamentos disponíveis para essas arboviroses ainda são bastante limitados, restringindo-se ao controle dos sintomas. Atualmente, apenas a vacina contra febre amarela é bem estabelecida ${ }^{5}$. Nesse contexto, as formas mais eficazes de mitigação dessas doenças são os controles mecânico, biológico e químico dos insetos vetores ${ }^{6}$.

O controle mecânico tem como principal objetivo eliminar criadouros, principalmente por ações de saneamento básico. Já o controle biológico pode ser implementado utilizando-se predadores ou patógenos capazes de reduzir a população vetorial ou a transmissão dos agentes etiológicos dessas doenças. Um destaque de controle biológico é o uso da bactéria Bacillus thuringienses israelensis (Bti), que apresenta relevante atividade larvicida ${ }^{6}$. Uma forma inovadora de controle é a infecção de Ae. aegypti com bactérias do gênero Wolbachia, que proporcionam redução na transmissão dos arbovírus pelo mosquito ${ }^{7}$.

Outra técnica de controle biológico envolve modificações genéticas do mosquito macho. A alteração é capaz de reduzir a população de mosquitos selvagens, ao gerar proles que não atingem a fase adulta. Apesar de promissora, a iniciativa ainda é incipiente e deve ser praticada de forma complementar a outras vertentes de controle do vetor ${ }^{8}$.

O controle químico é realizado por meio do uso de inseticidas para as diferentes fases de desenvolvimento do Ae. aegypti, sendo a maior parte piretroides ${ }^{9}$. Compostos repelentes de ambiente e tópicos também são aplicados para evitar contato com o mosquito vetor ${ }^{1}$.

Dentre as alternativas supracitadas, a mais utilizada é o controle químico, portanto, inseticidas merecem destaque quanto aos aspectos relacionados à sua regulação e ao impacto ambiental.

0 uso intensivo de pesticidas permite a seleção, ao longo do tempo, de insetos resistentes ${ }^{9,10,11}$. Um exemplo está na aplicação de temefós, organofosforado que foi utilizado como única forma de controle de larvas de Ae. aegypti durante mais de três décadas no Brasil, e hoje não apresenta efetividade contra o vetor ${ }^{9}$. A recomendação da Organização Mundial de Saúde (OMS) para se reduzir o desenvolvimento de resistência aos inseticidas disponíveis é a combinação de produtos com diferentes mecanismos de ação, e que possuam, como alvo, diferentes fases de desenvolvimento do mosquito ${ }^{12}$.

Inseticidas são poluentes terrestres, comumente detectados no meio ambiente, devido ao uso extensivo não só para controle de vetores envolvendo saúde pública, como também na produção agrícola. Organoclorados, como o dicloro-difenil-tricloroetano (DDT), apesar de terem sido banidos em muitos países, são os pesticidas encontrados em maior concentração no solo $\mathrm{e}$ na água em virtude de sua lenta biodegradabilidade ${ }^{13,14}$. Outro prejuízo ambiental referente ao uso de inseticidas está relacionado aos efeitos em organismos não alvo, como animais aquáticos, répteis, anfíbios, plantas e polinizadores ${ }^{15}$. Não só os inseticidas ambientais, mas aqueles aplicados na pele apresentam risco ao meio ambiente ${ }^{16}$. Avaliar os potenciais prejuízos para os organismos não alvo, bem como a persistência no ambiente, faz parte do processo de registro de um produto para que este seja seguro para seres humanos, e para os ecossistemas onde serão inseridos ${ }^{17}$. Neste contexto, é importante promover o desenvolvimento e a regulação de novas formulações, eficazes, seguras e sustentáveis.

Tendo em vista que as normas aplicadas ao registro e à análise de inseticidas no país estão esparsas em diferentes órgãos públicos, este artigo traz, de maneira didática, os aspectos regulatórios necessários à submissão de registro de inseticidas e repelentes no Brasil, à luz das orientações da OMS.

Adicionalmente, foi realizado um levantamento comparativo dos ingredientes ativos para controle do Ae. aegypti aprovados nos Estados Unidos da América - EUA (pela United States Environmental Protection Agency - EPA), na União Europeia UE (pela European Chemicals Agency - ECHA) e no Brasil (pela Agência Nacional de Vigilância Sanitária - Anvisa). Assim, objetivou-se entender se possíveis divergências regulatórias frente às orientações internacionais podem impactar no registro de produtos. Por fim, considerando o potencial impacto ambiental do uso de inseticidas, apresentamos um panorama geral dos estudos ecotoxicológicos dos ingredientes ativos comercializados nos três países, com levantamento quantitativo no banco de dados ECOTOX Knowledgebase. 


\section{MÉTODO}

Foi realizada uma análise de documentos sobre os requisitos necessários para o registro de inseticidas e repelentes para controle do Ae. aegypti no Brasil, incluindo-se a legislação brasileira vigente e as recomendações da OMS. Foi avaliado, ainda, o panorama geral dos ingredientes ativos registrados nos EUA e no Brasil, uma vez que os marcos regulatórios estabelecidos pela Anvisa orientam que sejam adotadas as recomendações da OMS ou EPA para o desenvolvimento dos produtos. Foram também consultados os ingredientes ativos disponíveis na UE para controle do vetor, de forma comparativa entre os três países.

Tendo em vista que a EPA é a única agência regulatória, das três discutidas neste trabalho, que disponibiliza um sistema de busca de produtos aprovados para controle específico de Aedes, tomou-se como base os ingredientes ativos obtidos em sua base de dados National Pesticide Information Center (NPRO). Ao resultado dessa pesquisa, foram somados outros ingredientes orientados pela OMS e pelo Centers for Disease Control and Prevention $(C D C)$ para controle do mosquito. A partir daí, verificou-se a autorização destes ingredientes pela Anvisa e pela ECHA.

As informações relacionadas aos produtos utilizados no Brasil foram obtidas por meio de notas oficiais do Portal da Anvisa, bem como da Ouvidoria da Agência e documentos do Ministério da Saúde (MS). Aqueles utilizados nos EUA foram disponibilizados pelo NPRO (inseticidas ambientais), EPA e CDC (repelentes tópicos). Os ingredientes ativos disponíveis na UE foram obtidos a partir de dados da ECHA (grupos PT18 e PT19 que incluem inseticidas de ambiente e repelentes) e European Centre for Disease Prevention and Control (ECDC).

Um panorama quantitativo de estudos ecotoxicológicos dos ingredientes ativos em organismos aquáticos e terrestres foi obtido a partir de consulta ao banco de dados ecotoxicológicos ECOTOX Knowledgebase ${ }^{18}$. Os ativos de inseticidas foram pesquisados pelo Chemical Abstract Service (CAS) ou nome científico, em caso de organismos. Adicionalmente, foram abordados estudos ecotoxicológicos de inseticidas disponíveis na literatura.

\section{RESULTADOS}

\section{Recomendações da OMS para o registro de inseticidas}

De acordo com a OMS, inseticidas devem ser submetidos a análises de eficácia no organismo-alvo e a análises toxicológicas para investigar se o produto é seguro para a saúde humana, os animais e o meio ambiente. Garantir que os produtos registrados sejam eficazes e seguros são o principal objetivo das agências reguladoras em todo o mundo. Desse modo, a empresa interessada em registrar o pesticida deve apresentar os documentos que comprovem a sua eficácia e proporcionem instrumentos para que a agência reguladora faça uma análise de risco e proponha exigências de manejo do produto e seus resíduos. Após análise, a depender dos critérios de cada agência, a solicitação de registro pode ser deferida de forma total ou provisória, com restrições ou condições, ou ser indeferida, quando o produto não se apresentar eficaz ou seguro ${ }^{17}$.
Os estudos apresentados no dossiê de registro devem ser de alta qualidade, confiabilidade e, sempre que possível, baseados em padrões e protocolos de organizações internacionais, como Organização das Nações Unidas para Agricultura e Alimentação (FAO), OMS e Organização para a Cooperação e Desenvolvimento Econômico (OCDE). Informações importantes que devem constar no dossiê são: (A) identidade e características físico-químicas do ingrediente ativo e sua respectiva formulação; (B) métodos analíticos; (C) toxicidade humana e ambiental; (D) uso e rotulagem proposta; (E) ficha de segurança do produto; (F) comprovação de eficácia para o uso pretendido (avaliada contra o vetor e, adicionalmente, sob as condições ecológicas do meio em que se pretende aplicar o produto); (G) resíduos gerados pelo uso e aplicação do produto; (H) manejo das embalagens; (I) disposição de resíduos. Todavia, as exigências podem variar de acordo com as características do produto, como a natureza do pesticida e sua escala de uso ${ }^{17}$.

Existem guias internacionais que estabelecem critérios para os estudos de eficácia de pesticidas. No caso de produtos para controle de vetores de doenças com impacto na saúde pública, utilizam-se os critérios estabelecidos pelo Esquema de Avaliação de Pesticidas da OMS (Pesticide Evaluation Scheme - WHOPES) ${ }^{19}$. Além de comprovar eficácia, a empresa deve apresentar informações de toxicidade humana e ambiental de acordo com a exposição decorrente da forma de aplicação e do uso do produto. Os estudos de segurança em humanos podem englobar toxicidade oral aguda, dérmica, por inalação; irritação dérmica e ocular; sensibilização dérmica; toxicidade por administração repetida (subaguda à crônica); toxicidade reprodutiva e de desenvolvimento; além de estudos de carcinogenicidade, genotoxicidade e relacionados ao metabolismo de plantas e animais ${ }^{17}$.

O perfil de ecotoxicidade deve ser baseado em estudos de toxicidade em organismos não alvo aquáticos e terrestres também apropriados ao modo de uso pretendido para o pesticida. Informações sobre persistência e bioacumulação do ingrediente ativo e da formulação no meio ambiente também são necessárias ${ }^{17}$. Existem diversos protocolos disponibilizados pela OCDE em diferentes organismos não alvo para os experimentos $\operatorname{citados}^{20}$.

Tendo em vista a grande relevância associada ao desenvolvimento de resistência dos vetores, a autoridade competente deve avaliar o risco potencial de desenvolvimento de resistência no momento do registro. Para isso, a empresa interessada deve disponibilizar, por exemplo: casos de resistência já relatados para compostos semelhantes ${ }^{17}$.

Panorama geral do uso e registro de inseticidas e repelentes no Brasil

O registro de inseticidas no Brasil está amparado principalmente por três instituições federais: o Ministério da Agricultura, Pecuária e Abastecimento (MAPA); o Ministério do Meio Ambiente (MMA) e o MS. Segundo o Decreto $n^{\circ} 4.074$, de 4 de janeiro de $2002^{21}$, cabe ao MAPA, ao MMA e ao MS conceder o registro de agrotóxicos, produtos técnicos, pré-misturas e afins. Contudo, cada uma dessas instituições fica responsável pela análise técnica das solicitações de registro dos produtos de acordo com a 
sua expertise de atuação. O MS, representado pela Anvisa, conforme Decreto $n^{\circ} 3.029$, de 16 de abril de 199922, que aprova seu regulamento, é responsável por conceder o registro de produtos destinados ao uso em ambientes urbanos, industriais, domiciliares, públicos ou coletivos, ao tratamento de água e ao uso em campanhas de saúde pública, como é o caso de inseticidas utilizados para o controle do Ae. aegypti ${ }^{21}$. O MMA fica incumbido de fazer a avaliação ambiental dos produtos destinados à saúde pública registrados e estabelecer sua periculosidade. Neste caso, não há participação do MAPA no registro ${ }^{21}$. Está, ainda, entre as atribuições da Anvisa, o registro de repelentes de insetos para aplicação na pele, que são classificados como produtos cosméticos pela instituição ${ }^{23}$.

\section{Registro de inseticidas de ambiente no Brasil}

Produtos saneantes destinados ao controle de vetores relacionados à saúde pública devem ser registrados em concordância com as exigências das Resoluções de Diretoria Colegiada (RDC) $n^{\circ} 34$, de 16 de agosto de $2010^{24}$ e no 59, de 17 de dezembro de $2010^{25}$, da Anvisa. Nessa categoria, estão englobados produtos inseticidas de ambiente, também denominados repelentes ambientais pela agência ${ }^{26}$.

Os produtos saneantes podem ter três funções, segundo definições da RDC n59/2010, que estabelece os procedimentos e requisitos técnicos para a notificação e o registro de produtos saneantes: (I) desinfecção, (II) desinfestação ou (III) desodorização. Com base nas definições, inseticidas são classificados como desinfestantes - que matam, inativam ou repelem organismos indesejáveis no ambiente, sobre objetos, superfícies inanimadas ou em plantas ${ }^{25}$.

De acordo com a resolução supracitada, desinfestantes são classificados como de risco II e, por isso, devem ser registrados na Anvisa. O relatório técnico do produto, submetido para o registro, deve conter: categoria (inseticida ou repelente), destino/ aplicação, composição qualitativa e quantitativa, denominação e características químicas, modo de ação, embalagens, sistema de identificação de lote, metodologia de análise do ingrediente ativo, grau de pureza dos ingredientes, identidade/concentração/toxicidade das impurezas, modo e restrições de uso, forma de apresentação, incompatibilidades físico-químicas, pragas contra as quais é indicado, dados toxicológicos, avaliação de risco, estudos de eficácia, estudos de estabilidade bem como de destinação final do produto ${ }^{25}$. Os limites de toxicidade aceitáveis estão descritos na Resolução n ${ }^{\circ} 34 / 2010^{24}$, de acordo com o estipulado pela $\mathrm{OMS}^{27}$.

De acordo com a Anvisa, em produtos desinfestantes, apenas é permitido o uso de ativos que possuem monografias aprovadas pela agência. Em se tratando de ingrediente ativo não autorizado previamente, ou seja, de caráter inovador no país, uma das medidas iniciais para o registro é a apresentação de dados toxicológicos dispostos no apêndice IV da Resolução n $\mathrm{n}^{\circ} 34 / 2010^{24}$ que, mediante aprovação, culminarão na publicação da monografia no portal da agência ${ }^{28}$. A monografia apresenta, além dos dados toxicológicos, o nome comum e químico do ingrediente ativo, classe, indicação de uso e limites máximos de resíduo ${ }^{29}$.
A OMS recomenda seis inseticidas, aprovados no âmbito da saúde pública, para serem utilizados no controle de mosquitos vetores adultos. Desses, cinco pertencem à classe dos piretroides - deltametrina, lambda-cialotrina, permetrina, aletrina, cifenotrina - e o outro é a malationa (organofosforado) ${ }^{30}$. A maior parte dos inseticidas registrados é à base de piretrinas ou piretroides ${ }^{26}$. Porém, a malationa tem sido o inseticida adotado para controle dos adultos, em razão da resistência apresentada pelo vetor a piretroides no país ${ }^{31,32}$. Dentre os larvicidas utilizados, têm-se: piriproxifem ${ }^{33}$, metopreno ${ }^{29}$, óleo de neem (azadiractina) ${ }^{29,34}$, diflubenzurom ${ }^{33}$, novalurom ${ }^{35}$ Bti $^{32}$ e espinosade ${ }^{36}$.

\section{Registro de repelentes tópicos no Brasil}

Os repelentes de insetos aplicados topicamente são enquadrados, para fins de registro, como produtos cosméticos e devem observar requisitos técnicos da RDC $n^{\circ} 19$, de 10 de abril de $2013^{23}$. De acordo com essa resolução, para registro de repelentes de insetos, deve-se comprovar sua segurança e eficácia. Os dados devem incluir irritação cutânea primária e acumulada, sensibilização cutânea e fotossensibilização. Para eficácia, devem ser utilizadas as diretrizes estipuladas pela EPA, pela OMS, ou por outras metodologias reconhecidas internacionalmente ${ }^{22,37}$.

O protocolo da OMS sobre avaliação da eficácia de repelentes utilizados na pele estabelece que sejam determinados, inicialmente em testes laboratoriais, a dose efetiva e o tempo de proteção fornecido pelo produto em avaliação. Nesse estágio, determina-se a curva dose-resposta, além das doses responsáveis por $50,00 \%$ e $99,90 \%$ do efeito. Como controle positivo, utiliza-se o N,N-dietil-meta-toluamida (DEET) ${ }^{38}$.

No Brasil, existem repelentes comercializados com os ativos DEET, icaridina, IR3535 e óleo de citronela eficazes contra o Ae. aegypti, segundo alerta da Anvisa $n^{\circ} 3.032$ sobre o uso de repelentes e aplicação de inseticidas ${ }^{26}$ e questionamento via Ouvidoria da Agência ${ }^{39}$. DEET é o repelente mais utilizado no mundo todo, com excelente perfil de segurança. Nos guias de proteção contra o zika vírus, o DEET tem sido o repelente de escolha, inclusive por gestantes ${ }^{40,41}$.

Panorama geral do registro de inseticidas de ambiente e repelentes tópicos nos EUA

Segundo a EPA, até o momento, existem oito diferentes ingredientes ativos registrados nos EUA como repelentes de aplicação tópica contra mosquitos. Dentre eles, estão o óleo de Nepeta cataria - catmint (quatro produtos); óleo de citronela (quatro produtos); DEET (mais de 500 produtos); IR3535 (45 produtos); óleo de eucalipto limão (13 produtos); para-mentano-diol (PMD) (oito produtos); icaridina (40 produtos); e a 2-undecanona (um produto $)^{41,42,43}$. A recomendação do CDC é de que sejam utilizados os ativos DEET, icaridina, IR3535, óleo de eucalipto limão, PMD (forma sintética do óleo de eucalipto limão) ou 2-undecanona para proteção específica contra o Ae. aegypti ${ }^{44}$.

Como larvicidas, são utilizados Bti, diflubenzurom, espinosade, metopreno, novalurom e piriproxifem além de filmes e óleos colocados na superfície da água, capazes de evitar trocas gasosas 
entre atmosfera e o ambiente aquático ${ }^{37}$. Contudo, no NPRO foram encontrados apenas os ativos Bti, metopreno e piriproxifem com indicação específica para controle de Ae. aegypti ${ }^{45}$, apesar de todos os demais ingredientes citados pelo $\mathrm{CDC}^{46}$ serem registrados na agência americana ${ }^{45}$.

Em pesquisa por pesticidas utilizados para controle de Ae. aegypti no NPRO, foram encontrados 80 produtos ativos, em sua maioria constituídos por piretrinas e piretroides. Dentre os ingredientes ativos listados estão: permetrina, praletrina, fenotrina, metoflutrina, esfenvalerato, piriproxifem, lambda-cialotrina, metopreno e o fungo Beauveria bassiana ${ }^{38}$. Os piretroides aletrina e cifenotrina, e o organofosforado malationa, indicados pela OMS para

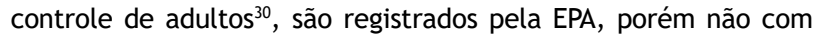
indicação específica para Ae. aegypti, em consulta ao $\mathrm{NPRO}^{45}$.

Panorama geral do registro de inseticidas de ambiente e repelentes tópicos na UE

Produtos destinados à proteção de seres humanos, animais, materiais ou artigos contra organismos como pestes ou bactérias são denominados como biocidas pela UE. 0 registro destes produtos é descrito pela regulação Biocidal Products Regulation (BPR, Regulation (EU) 528/2012). As substâncias ativas presentes em biocidas devem estar, como regra geral, previamente aprovadas pela $\mathrm{ECHA}^{47}$. As substâncias ativas aprovadas pela agência para controle do mosquito podem estar enquadradas como (PT18) inseticidas, acaricidas e produtos para controle de outros artrópodes, ou (PT19) repelentes e atrativos. Considerando estes dois grupos, existem 88 substâncias ativas aprovadas pela EU ${ }^{48}$. Contudo, destes ativos, não se pode mensurar pelo sistema de busca quantos são indicados para o controle especificamente de mosquitos.
Dos inseticidas utilizados para controle de Ae. aegypti (Quadro), quatro não são aprovados pela UE (ECHA): esfenvalerato, que teve sua autorização descontinuada, novalurom, malationa e Beauveria bassiana. Aletrina está sob análise pela agência ${ }^{49}$.

No que se refere a repelentes tópicos, a ECDC indica que sejam seguidas as orientações do CDC acerca de repelentes para proteção contra picadas pelo Ae. aegypti ${ }^{49}$. DEET, IR3535 e 2-undecanona estão aprovados na UE, enquanto PMD, óleo de eucalipto limão e icaridina estão sob avaliação pela ECHA ${ }^{48}$.

Estudos ecotoxicológicos dos ingredientes ativos presentes em produtos para controle de Ae. aegypti

Os pesticidas são desenvolvidos para proteger alimentos e/ou a saúde. Contudo, seus mecanismos de ação envolvem matar, repelir, prevenir ou ameaçar insetos, o que, inevitavelmente, apresenta certa toxicidade para o meio ambiente ${ }^{50}$. Os ecossistemas aquáticos, por serem depósito final de resíduos, são diretamente contaminados. Modelos de avaliação de toxicidade aguda e crônica de pesticidas nesses ecossistemas envolvem organismos de diferentes níveis tróficos, envolvendo microcrustáceos, como Daphnia magna, Ceriodaphnia dubia, Artemia salina e Dendrocephalus brasiliensis; peixes, como Xiphophorus maculatus e Danio rerio (zebrafish); e crustáceos, como Eurytemora affinis e Leander tenuicornis ${ }^{51,52,53,54}$.

Os efeitos ecotoxicológicos podem ser avaliados também em plantas, por meio de estudos de fitotoxicidade, e em animais que interagem diretamente com plantas, como por exemplo os polinizadores $^{50}$. Esse conjunto de estudos é utilizado tanto para avaliar o risco ecotoxicológico de determinado produto, quanto para redefinir doses efetivas e ambientalmente seguras a serem aplicadas ${ }^{51}$.

Quadro. Inseticidas de ambiente utilizados no controle de Ae. aegypti (Brasil, EUA, UE).

\begin{tabular}{|c|c|c|c|c|}
\hline $\begin{array}{l}\text { Chemical Abstract } \\
\text { Service }\end{array}$ & Ingrediente ativo & $\begin{array}{l}\text { States Environmental } \\
\text { Protection Agency }\end{array}$ & $\begin{array}{c}\text { Agência Nacional de } \\
\text { Vigilância Sanitária }\end{array}$ & European Chemicals Agency \\
\hline $584-79-2$ & aletrina $^{\mathrm{a}}$ & autorizado & autorizado & em análise \\
\hline $63428-82-0$ & Beauveria bassiana ${ }^{\mathrm{b}}$ & autorizado & autorizado & não há registro \\
\hline$N A^{c}$ & $B t i^{c, d}$ & autorizado & autorizado & autorizado \\
\hline $39515-40-7$ & cifenotrina $^{\mathrm{a}}$ & autorizado & autorizado & autorizado \\
\hline $35367-38-5$ & diflubenzuromm ${ }^{c, d}$ & autorizado & autorizado & autorizado \\
\hline $66230-04-4$ & esfenvalerato ${ }^{b}$ & autorizado & autorizado & descontinuado \\
\hline $168316-95-8$ & espinosade ${ }^{c}$ & autorizado & autorizado & autorizado \\
\hline $26002-80-2$ & fenotrina $^{b}$ & autorizado & autorizado & autorizado \\
\hline $91465-08-6$ & lambda-cialotrina $a^{\mathrm{a}, \mathrm{b}}$ & autorizado & autorizado & autorizado \\
\hline $121-75-5$ & malationa $^{\mathrm{a}}$ & autorizado & autorizado & não há registro \\
\hline $240494-70-6$ & metoflutrina ${ }^{b}$ & autorizado & autorizado & autorizado \\
\hline $40596-69-8$ & metopreno $0^{\mathrm{b}, \mathrm{c}}$ & autorizado & autorizado & autorizado \\
\hline $116714-46-6$ & novalurom ${ }^{c, d}$ & autorizado & autorizado & não há registro \\
\hline $11141-17-6$ & óleo de neem (azadiractina) ${ }^{d}$ & autorizado & autorizado & autorizado \\
\hline $52645-53-1$ & permetrina $a^{a, b}$ & autorizado & autorizado & autorizado \\
\hline $8003-34-7$ & piretrinas e piretroides $^{\mathrm{b}}$ & autorizado & autorizado & autorizado \\
\hline $95737-68-1$ & piriproxifem ${ }^{\mathrm{b}, \mathrm{c}, \mathrm{d}}$ & autorizado & autorizado & autorizado \\
\hline $23031-36-9$ & praletrina $^{\mathrm{a}, \mathrm{b}}$ & autorizado & autorizado & autorizado \\
\hline
\end{tabular}

Fonte: Organização Mundial da Saúde ${ }^{26}$; Environmental Protection Agency (EPA) ${ }^{41}$; Centers for Disease Control and Prevention (CDC) ${ }^{42}$; European Centre for Disease Prevention and Control (ECDC) ${ }^{49}$; Agência Nacional de Vigilância Sanitária ${ }^{28,29,30,31}$.

${ }^{a}$ Indicado pela Organização Mundial da Saúde ${ }^{26}$; ${ }^{b}$ Registrado na Environmental Protection Agency (EPA) ${ }^{41}$; ${ }^{c}$ Indicado pelo Centers for Disease Control and Prevention (CDC ${ }^{42}$; ${ }^{d}$ Indicado pela Agência Nacional de Vigilância Sanitária ${ }^{28,29,30,31}$; NA: não se aplica; Bti: Bacillus thuringienses israelensis. 
A presença de resíduos de inseticidas já foi detectada em solos e sedimentos em diversas regiões do mundo ${ }^{50}$, bem como em ambientes aquáticos, onde já se observou inclusive que variações no $\mathrm{pH}$ da água podem influenciar diretamente a dissipação dos inseticidas ${ }^{55}$.

Estudos com o piriproxifem, ativo comumente utilizado em produtos para controle de larvas de Ae. aegypti, apontam efeitos tóxicos para alguns microcrustáceos ${ }^{51}$, peixes e crustá$\operatorname{ceos}^{52,56}$. Diflubenzurom, amplamente utilizado em substituição ao temefós devido à resistência deste, apresentou alta toxicidade para Daphnia magna ${ }^{57}$. Ambos, diflubenzuron e temefós, foram considerados tóxicos para os peixes Oreochromis niloticus e Hyphessobrycon eques ${ }^{58}$. Outro estudo observou que temefós e diflubenzurom reduziram populações de insetos aquáticos ${ }^{59}$. Piretroides sintéticos, utilizados para controle de mosquitos adultos, apesar de serem considerados de baixa toxicidade para mamíferos, apresentaram alta toxicidade para peixes, camarões e lagosta ${ }^{60,61}$. Organofosforados e carbamatos, tidos como menos persistentes no ambiente e menos tóxicos para mamíferos que organoclorados, afetam a reprodução de $D$. magna, bem como o comportamento de répteis ${ }^{62}$.

Apesar de estudos em anfíbios serem bastante escassos, observou-se que o DDT é acumulado em Xenopus sp. Contudo, o potencial de acumulação de outros pesticidas em sapos é desconhecido. Detectou-se que malationa e alguns piretroides apresentaram toxicidade para este modelo ${ }^{15}$. Um estudo considerou metopreno e Bti como inseticidas de baixo risco para organismos não alvo com base nas concentrações detectadas no ambiente ${ }^{63}$. Porém, um alerta da UE apontou riscos para pássaros após aplicação repetida de $\mathrm{Bti}^{64}$.

DEET, repelente tópico utilizado por mais de 30 anos e considerado seguro para a população em geral, já foi reportado em estações de tratamento de água e no mar. Apesar disso, a toxicidade aguda e crônica em organismos aquáticos é improvável, baseando-se nas concentrações encontradas no ambiente ${ }^{65}$.

Mamíferos também são afetados secundariamente pelos inseticidas, devido a sua semelhança fisiológica com insetos. Considerando que o mecanismo de ação desses produtos afeta em geral o sistema nervoso de insetos, como receptores nicotínicos de acetilcolina, acetilcolinesterase, receptores de ácido gama-aminobutírico (GABA) e canais de sódio voltagem-dependentes, o sistema nervoso de mamíferos também pode ser afetado ${ }^{66}$.

Considerando que os dados expostos ratificam a necessidade de se avaliar o risco ambiental em organismos não alvo associado ao uso de inseticidas, realizou-se um levantamento quantitativo dos estudos ecotoxicológicos disponíveis no banco de dados ECOTOX Knowledgebase para estes produtos. Foram encontrados 32.717 estudos para ingredientes de inseticidas de ambiente (Figura 1), sendo 14.354 em organismos aquáticos e $18.363 \mathrm{em}$ organismos terrestres. Dos 18 ingredientes pesquisados, oito são piretrinas ou piretroides (aletrina, cifenotrina, fenotrina, lambda-cialotrina, metoflutrina, permetrina, praletrina, piretrinas e piretroides). Cerca de $41,00 \%$ dos estudos ecotoxicológicos encontrados são referentes a estas classes de produtos, sendo a metoflutrina o ativo com o menor número de estudos disponíveis da classe, aproximadamente $0,01 \%$ do total de estudos. Dois ingredientes, Bti e Beauveria bassiana, não têm nenhum estudo ecotoxicológico na base de dados. A proporção de estudos em organismos aquáticos e terrestres mostrou-se equilibrada, $44,00 \%$ e $56,00 \%$, respectivamente ${ }^{18}$.

0 número de estudos toxicológicos para repelentes tópicos é significativamente menor (Figura 2), com no máximo 357 por ingrediente ativo, nesse caso o DEET. Foi encontrado um total de 438 estudos para sete compostos utilizados como repelentes tópicos, considerando investigações em organismos não alvo terrestres e aquáticos ${ }^{18}$.

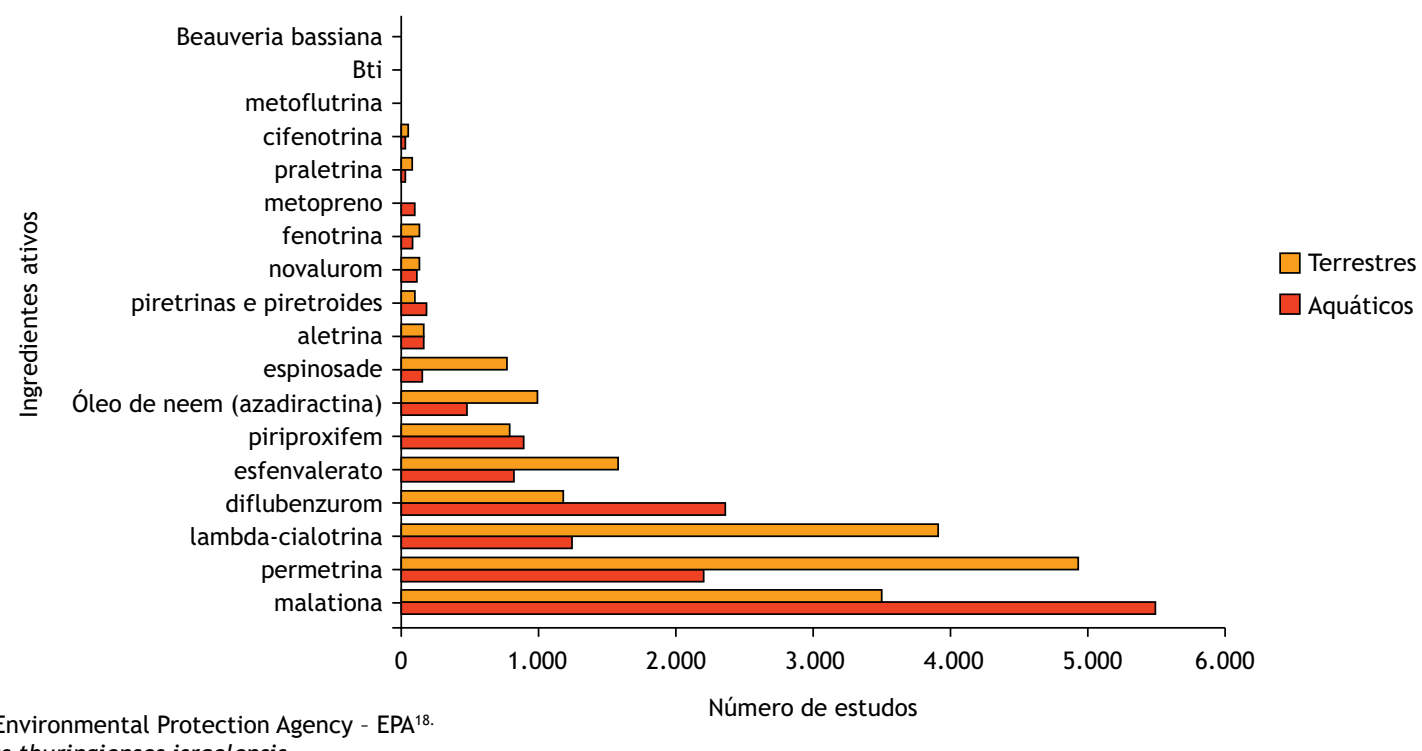

Fonte: US Environmental Protection Agency - EPA ${ }^{18}$ Bti: Bacillus thuringienses israelensis.

Figura 1. Estudos ecotoxicológicos em organismos não alvo disponíveis no ECOTOX Knowledgebase por ingrediente ativo de inseticidas de ambiente. 


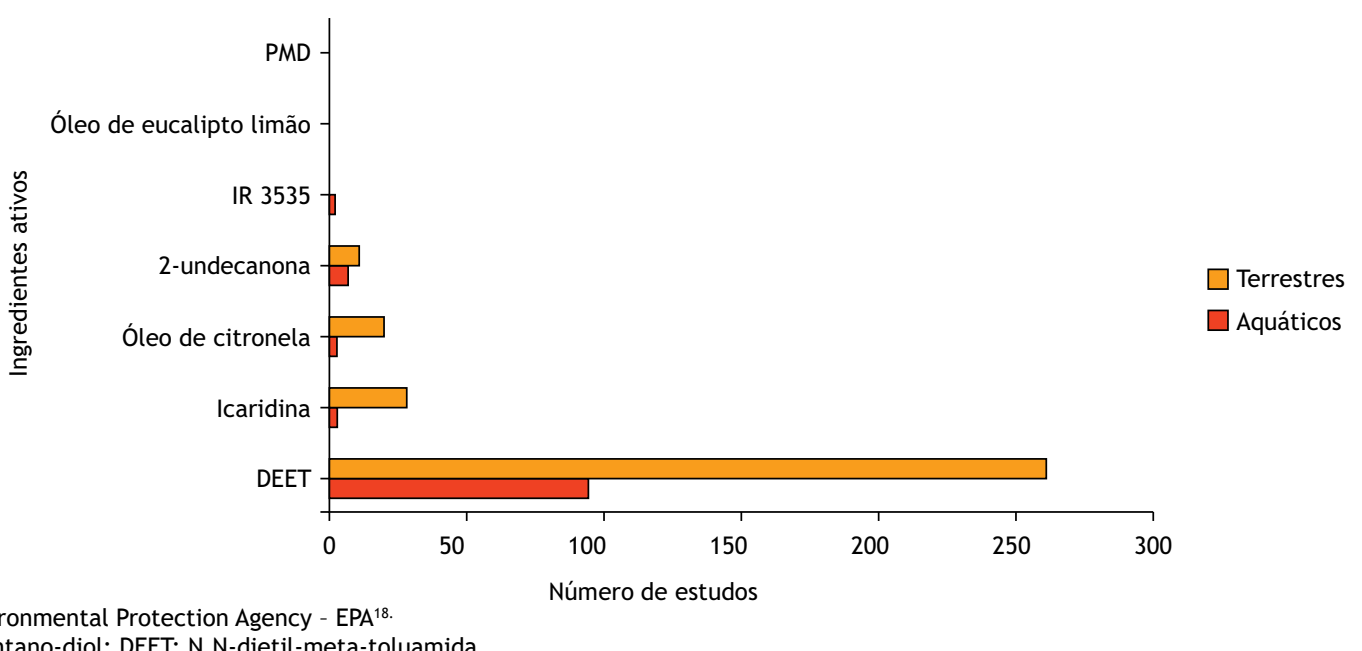

Fonte: US Environmental Protection Agency - EPA 18.

PMD: para-mentano-diol; DEET: N,N-dietil-meta-toluamida.

Figura 2. Estudos ecotoxicológicos em organismos não alvo disponíveis no ECOTOX Knowledgebase por ingrediente ativo de repelentes tópicos.

\section{DISCUSSÃO}

O registro de inseticidas de ambiente e de repelentes tópicos está a cargo da Anvisa no Brasil, com análise de periculosidade ambiental realizada pelo Ibama ${ }^{21,23,24,25}$. As resoluções estabelecidas pela Anvisa estão de acordo com as orientações para registro de pesticidas da OMS ${ }^{17}$, inclusive os limites de toxicidade aceitáveis ${ }^{17,24}$.

A grande maioria dos produtos inseticidas de ambiente registrados nos EUA é a base de piretroides, assim como no Brasil. Como consequência dessa limitação de ingredientes ativos disponíveis para controle vetorial, observa-se o rápido e esparso desenvolvimento de resistência pelos insetos. O Quadro traz um panorama geral comparativo dos inseticidas de ambiente para controle de Ae. aegypti utilizados nas regiões em destaque neste trabalho. Todos os ingredientes ativos presentes em produtos aprovados pela EPA para controle ambiental de Ae. aegypti, assim como os indicados pela $\mathrm{OMS}^{30}$ e pelo $\mathrm{CDC}^{46}$, possuem monografia aprovada pela Anvisa. Infere-se, portanto, que os critérios de avaliação para registro desses ingredientes ativos nos dois países, EUA e Brasil, estejam alinhados. 0 óleo de neem (azadiractina) é indicado pela Anvisa para controle do vetor, apesar de não estar nas recomendações desses órgãos internacionais ${ }^{30,46}$. Há, contudo, registro desse ingrediente nas agências EPA ${ }^{45}$ e ECHA ${ }^{48}$; na EPA sem indicação explícita contra Ae. aegypti. A UE, por sua vez, mostrou-se mais restritiva quanto à autorização de inseticidas. Quatro ativos autorizados nos EUA e no Brasil não o são pela ECHA.

Apesar de os ingredientes ativos de inseticidas terem monografias aprovadas pela Anvisa, isso não garante que existam produtos comercializados com eles. Para o registro de novos produtos no Brasil, é necessário que as empresas protocolem as solicitações na Anvisa, visto que a agência não desempenha um papel ativo neste aspecto. Com a monografia aprovada para o ativo, a empresa pode protocolar a solicitação de registro para o produto contendo aquele ativo, juntamente com os demais requisitos exigidos pela legislação.
Com relação a repelentes tópicos, todos os indicados pelo $\mathrm{CDC}^{44}$ são autorizados pela agência americana ${ }^{43}$, ao passo que no Brasil, três não constam em indicações da Anvisa e, na UE, três ainda estão sob análise para registro ${ }^{48}$. 0 óleo de citronela, apesar de indicado no alerta da $A_{n v i s a}{ }^{26}$, não é orientado pelo CDC como forma de proteção contra o Ae. aegypti ${ }^{44}$. O CDC indica outros três ingredientes para proteção contra picadas do vetor, que não constam nas orientações da Anvisa: óleo de eucalipto limão, PMD e 2-undecanona ${ }^{44}$. Foi observada uma maior divergência quanto à indicação de repelentes tópicos pelas agências e CDC, comparando-se com dados obtidos para os inseticidas ambientais.

Considerando-se os ingredientes ativos aprovados pelas três agências, tanto de uso ambiental quanto tópico, a estadunidense tem o maior número de ativos registrados, seguido pela Anvisa e ECHA. Essa diferença sugere que a agência europeia disponha de critérios mais rígidos de registro.

É importante ressaltar que as diferenças entre os sistemas de busca das páginas eletrônicas das agências reguladoras resultaram em limitações para o presente estudo. O portal da Anvisa não dispõe de mecanismos de pesquisa por produtos (inseticidas e repelentes) registrados por ingrediente ativo ou indicação de uso. Logo, infere-se que o número de ingredientes ativos registrados dispostos neste estudo pode estar subestimado, tendo em vista que foi baseado em informações oficiais na página eletrônica da agência e questionamentos via ouvidoria. A ECHA, apesar de disponibilizar busca por ingredientes ativos e indicação de uso, não informa o organismo contra a qual a substância foi aprovada.

Com relação à toxicidade ambiental de inseticidas utilizados para controle de Ae. aegypti, observou-se que a disponibilidade de estudos na literatura, em geral, refere-se aos produtos de aplicação ambiental, como larvicidas e adulticidas. Esse achado foi reforçado pelos dados obtidos no ECOTOX Knowledgebase, em que o número absoluto de estudos para repelentes tópicos é expressivamente menor que os disponíveis para inseticidas ambientais. É importante reforçar que os produtos aplicados 
na pele alcançam diretamente efluentes, por meio da água do banho ou de resíduos espaciais da aplicação que atingem o solo, podendo afetar diversos organismos não alvo nesse percurso. 0 DEET, que é o mais utilizado dos produtos tópicos, foi considerado de baixo risco ambiental, devido a sua biodegradabilidade e aos baixos níveis encontrados em ambientes aquáticos. Contudo, esses são produtos passíveis de biotransformação e bioacumulação, podendo causar toxicidade para organismos não alvo ${ }^{16}$.

Observou-se que muitos dos inseticidas ambientais empregados no controle vetorial foram detectados em solos e água, além de estarem relacionados à toxicidade aguda e crônica em diferentes organismos não alvo. Existem poucas avaliações de ecotoxicidade que envolvam animais de diferentes níveis tróficos para um mesmo ativo. Estudos recentes apontam preocupação com a residualidade de produtos larvicidas em fase de desenvolvimento ${ }^{67}$, fator este que deve ser analisado em paralelo com a exposição crônica dos organismos presentes nos locais de aplicação.

Existe a necessidade de avaliações mais complexas para determinar o risco ambiental desses produtos, para que seja possível chegar a conclusões que culminem, por exemplo, na alteração dos padrões de uso quando os ativos ameaçarem o meio ambiente. Porém, não há uma clara definição nas normas nacionais e internacionais consultadas de quais estudos seriam suficientes para comprovação da segurança ambiental. Sugere-se que os tipos de teste exigidos para registro não sejam especificados em virtude da existência de diferentes classes de inseticidas, com mecanismos de ação e modo de utilização próprios. Assim, a agência que recebe a solicitação de registro é responsável por avaliar se os estudos apresentados são suficientes para garantir o uso seguro do produto.

Os riscos ambientais apresentados por alguns dos ingredientes ativos enfatizam a necessidade de mais avaliações ecotoxicológicas, principalmente para os ingredientes ativos que não apresentam quaisquer estudos na base de dados pesquisada, como, por exemplo, o Bti. Este larvicida apresenta inclusive posicionamentos sobre segurança ambiental divergentes na literatura. Metopreno e novalurom também chamaram atenção pelo baixo número de estudos, $114(0,30 \%)$ e $244(0,70 \%)$, respectivamente (Figura 1), apesar de já terem sido associados a riscos para alguns organismos não alvo.

\section{CONCLUSÕES}

Este trabalho apresenta uma revisão das exigências para registro de inseticidas e repelentes no Brasil, abordando orientações da OMS para tal. Nesse aspecto, concluiu-se que as normas brasileiras estão condizentes com os requisitos internacionais. Contudo, observou-se a falta de guias pontuais sobre o registro de inseticidas inovadores no país, visto que há necessidade de que sejam elaboradas monografias de novos ingredientes ativos, com exigências específicas, previamente ao registro de produtos que os contenham.

Com relação à análise comparativa dos ingredientes ativos disponíveis no Brasil, EUA e UE, a ECHA (UE) mostrou-se a agência mais restritiva quanto à autorização de inseticidas e repelentes. A forma de obtenção dos dados variou de acordo com as ferramentas de busca disponibilizadas pelas agências, sendo essa uma limitação do estudo.

Por fim, o artigo mostra a necessidade de estudos ecotoxicológicos mais abrangentes, que incluam diferentes organismos não alvo para todos os inseticidas utilizados no controle vetorial. Observou-se que a maioria dos inseticidas apresenta certa toxicidade para os ecossistemas em que são inseridos. Ainda assim, alguns dos ingredientes ativos comercializados não têm estudos ecotoxicológicos ou há poucos disponíveis na base de dados pesquisada (ECOTOX Knowledgebase). Ressalta-se que, apesar de abrangente, a ECOTOX Knowledgebase possui algumas limitações. É provável que nem todos os estudos realizados estejam disponíveis nessa base de dados. Portanto, a não identificação de estudos ecotoxicológicos não significa que esses não foram realizados, apesar desse achado alertar para a necessidade de maior acesso à informação ou de realização de estudos. Em acréscimo, não foram identificadas ferramentas de busca para esse tipo de informação na página eletrônica da Anvisa. 0 acesso aos dados de impacto ambiental pode dar autonomia para o consumidor optar por produtos menos prejudiciais ao meio ambiente.

\section{REFERÊNCIAS}

1. Tabanca N, Bernier RU, Agramonte NM, Tsikolia M, Bloomquist JR. Discovery of repellents from natural products. Curr Org Chem. 2016;20(25):2690-702. https://doi.org/10.2174/1385272820666160421151503

2. Figueiredo R, Paiva C, Morato M. Arboviroses. Canal Saúde Fiocruz. 18 dez 2017.

3. Ministério da Saúde (BR). Monitoramento dos casos de dengue, febre de chikungunya e febre pelo vírus zika até a semana epidemiológica 49. Bol Epidemiol. 2016;47(38):1-10.

4. Ministério da Saúde (BR). Monitoramento dos casos de arboviroses urbanas transmitidas pelo Aedes (dengue, chikungunya e zika), semanas epidemiológicas 1 a 34. Bol Epidemiol. 2019;50(22):1-11.
5. Lindsay SW, Wilson A, Golding N, Scott TW, Takken W. Improving the built environment in urban areas to control Aedes egypti-borne diseases. Bull World Health Organ. 2017;95(8):607-8. https: / /doi.org/10.2471/BLT.16.189688

6. Zara ALDSA, Santos SMD, Fernandes-Oliveira ES, Carvalho RG, Coelho GE. Estratégias de controle do Aedes egypti: uma revisão. Epidemiol Serv Saude. 2016;25(2):391-404. https://doi.org/10.5123/s1679-49742016000200017

7. World Mosquito Program - WMP. WMP Brasil. Melbourne: World Mosquito Program; 2019[acesso 25 jul 2019]. Disponível em: http://www.eliminatedengue.com/brasil 
8. Seidlein L, Kekulé AS, Strickman D. Novel vector control approaches: the future for prevention of zika virus transmission? PLoS Med. 2017;14(1):1-3. https://doi.org/10.1371/journal.pmed.1002219

9. Bellinato DF, Viana-Medeiros PF, Araújo SC, Martins AJ, Lima JBP, Valle D. Resistance status to the insecticides temephos, deltamethrin, and diflubenzuron in brazilian Aedes egypti populations. Biomed Res Int. 2016;2016:1-13. https://doi.org/10.1155/2016/8603263

10. Liu N, Li M, Gong Y, Liu F, Li T. Cytochrome p450s: their expression, regulation, and role in insecticide resistance. Pestic Biochem Physiol. 2015;120:77-81. https://doi.org/10.1016/j.pestbp.2015.01.006

11. Vieira TB. Suscetibilidade e reversão da resistência a inseticidas em população de Aedes (stegomyia) aegypti (linnaeus, 1762) (diptera: culicidae) [dissertação]. Curitiba: Universidade Federal do Paraná; 2015.

12. World Health Organization - WHO. Monitoring and managing insecticide resistance in Aedes mosquito populations. Geneva: World Health Organization; 2016[acesso 12 ago 2019]. Disponível em: https://apps.who.int/iris/ bitstream/handle/10665/204588/WHO_ZIKV_VC_16.1_eng. pdf?sequence $=2$

13. World Health Organization - WHO. The use of DDT in malaria vector control: WHO position statement. Geneva: World Health Organization; 2011[acesso 6 out 2019]. Disponivel em: https://apps.who.int/iris/bitstream/ handle/10665/69945/WHO_HTM_GMP_2011_eng.pdf

14. Berg H, Manuweera G, Konradsen F. Global trends in the production and use of DDT for control of malaria and other vector-borne diseases. Malar J. 2017;16:1-8. https://doi.org/10.1186/s12936-017-2050-2

15. Wolmarans NJ, Bervoets L, Meire PWV. Current status and future prognosis of malaria vector control pesticide ecotoxicology and Xenopus sp. In: Voogt P, editor. Reviews of environmental contamination and toxicology volume 252 . Cham: Springer; 2019. p. 131-71.

16. Roy DN, Goswami R, Pal A. The insect repellents: a silent environmental chemical toxicant to the health. Environ Toxicol Pharmacol. 2017;50:91-102. https://doi.org/10.1016/j.etap.2017.01.019

17. World Health Organization - WHO. Guidelines for the registration of pesticides. Geneva: World Health Organization; 2010[acesso 5 jul 2019]. Disponível em: https://www.who.int/whopes/resources/ who_htm_ntd_whopes_2010.7/en/

18. US Environmental Protection Agency - EPA. Ecotox knowledgebase. Washington: United States Environmental Protection Agency; 2019[acesso 17 out 2019]. Disponível em: https://cfpub.epa.gov/ecotox/search.cfm

19. World Health Organization - WHO. Pesticides evaluation scheme. Geneva: World Health Organization; 2017[acesso 8 ago 2019]. Disponível em: https://www.who.int/ neglected_diseases/resources/WHOPES/en/

20. Organization for Economic Co-operation and Development OECD. Test guidelines for the chemicals. Paris: Organization for Economic Co-operation and Development; 2019[acesso 8 ago 2019]. Disponivel em: http://www.oecd.org/env/ehs/ testing/oecdguidelinesforthetestingofchemicals.htm

21. Brasil. Decreto $N^{\circ} 4.074$, de 4 de janeiro de 2002. Regulamenta a lei $\mathrm{N}^{\circ} 7.802$, de 11 de julho de 1989 , que dispõe sobre a pesquisa, a experimentação, a produção, a embalagem e rotulagem, o transporte, o armazenamento, a comercialização, a propaganda comercial, a utilização, a importação, a exportação, o destino final dos resíduos e embalagens, o registro, a classificação, o controle, a inspeção e a fiscalização de agrotóxicos, seus componentes e afins, e dá outras providências. Diário Oficial União. 8 jan 2002.

22. Brasil. Decreto $N^{\circ} 3.029$, de 16 de abril de 1999. Aprova o regulamento da agência nacional de vigilância sanitária, e dá outras providência. Diário Oficial União. 19 abr 1999.

23. Agência Nacional de Vigilância Sanitária - Anvisa. Resolução RDC N ${ }^{\circ} 19$, de 10 de abril de 2013. Dispõe sobre os requisitos técnicos para a concessão de registro de produtos cosméticos repelentes de insetos e dá outras providências. Diário Oficial União. 11 abr 2013.

24. Agência Nacional de Vigilância Sanitária - Anvisa. Resolução RDC $N^{\circ}$ 34, de 16 de agosto de 2010. Dispõe sobre o regulamento técnico para produtos saneantes desinfestantes. Diário Oficial União. 18 ago 2010.

25. Agência Nacional de Vigilância Sanitária - Anvisa. Resolução RDC N 59, de 17 de dezembro de 2010. Dispõe sobre os procedimentos e requisitos técnicos para a notificação e o registro de produtos saneantes e dá outras providências. Diário Oficial União. 22 dez 2010.

26. Agência Nacional de Vigilância Sanitária - Anvisa. Alertas e informes 3032: uso de repelentes e aplicação de inseticidas. Brasília: Agência Nacional de Vigilância Sanitária; 2019 [acesso 20 set 2019]. Disponível em: http://portal. anvisa.gov.br/anvisa-esclarece

27. World Health Organization - WHO. The WHO recommended classification of pesticides by Hazard. Geneva: World Health Organization; 2009[acesso 20 set 2019]. Disponivel em: https://www.who.int/ipcs/publications/pesticides_ hazard_2009.pdf

28. Agência Nacional de Vigilância Sanitária - Anvisa. Anvisa esclarece 2964: componentes proibidos e permitidos em saneantes. Brasília: Agência Nacional de Vigilância Sanitária; 2017[acesso 4 ago 2019]. Disponível em: http:// portal.anvisa.gov.br/anvisa-esclarece.

29. Agência Nacional de Vigilância Sanitária - Anvisa. Monografias autorizadas. Brasília: Agência Nacional de Vigilância Sanitária; 2019[acesso 15 jul 2019]. Disponível em : http://portal.anvisa.gov.br/registros-e-autorizacoes/ agrotoxicos/produtos/monografia-de-agrotoxicos/ autorizadas

30. World Health Organization - WHO. WHO recommended insecticides for space spraying against mosquitoes. Geneva: World Health Organization; 2012[acesso 15 jul 2019]. Disponivel em: http://www.hygiene-publique.gov.pf/IMG/ pdf/recommandations_oms_adulticides_moustiques_space_ spraying_2012.pdf 
31. Fundação Oswaldo Cruz - Fiocruz. Recomendação técnica sobre a interrupção do uso de inseticidas piretroides no controle do Aedes aegypti no Brasil. Rio de Janeiro: Fundação Oswaldo Cruz; 2011 [acesso 10 ago 2019]. Disponível em: http://www. fiocruz.br/ioc/media/NotaTecnica\%202\%20 IOC\%20v1\%20dengue\%206\%20set\%202011.pdf

32. Ministério da Saúde (BR). Diretrizes nacionais para a prevenção e controle de epidemias de dengue. Brasília: Ministério da Saúde; 2009[acesso 15 jul 2019]. Disponível em: http://bvsms.saude.gov.br/bvs/publicacoes/diretrizes_ nacionais_prevencao_controle_dengue.pdf

33. Agência Nacional de Vigilância Sanitária - Anvisa. Recomendações técnicas ao sistema nacional de vigilância sanitária para colaborar no combate ao Aedes aegypti e prevenção e controle da dengue, chikungunya e infecção pelo vírus zika. Brasília: Agência Nacional de Vigilância Sanitária; 2016[acesso 22 ago 2019]. Disponível em: http:// aprav.com.br/wp-content/uploads/2016/04/CartilhaANVISA-aedes-1.pdf

34. Agência Nacional de Vigilância Sanitária - Anvisa. Produtos saneantes para o combate ao Aedes aegypti. Brasília: Agência Nacional de Vigilância Sanitária; 2019[acesso 22 out 2019]. Disponível em: http://portal.anvisa.gov.br/ produtos-aedes

35. Ministério da Saúde (BR). Recomendações para uso do larvicida novaluron para controle de Aedes aegypti. Brasília: Ministério da Saúde; 2019[acesso 22 jul 2019]. Disponível em: http://portalarquivos2.saude.gov.br/images/pdf/2014/ abril/28/Instru----es-para-emprego-no-Novaluron.pdf

36. Prefeitura Municipal de Macaé. Prefeitura utiliza novo larvicida contra mosquito. Central de Noticias. 5 jun 2019[acesso 24 ago 2019]. Disponivel em: http://www. macae.rj.gov.br/index.php/noticias/leitura/noticia/ prefeitura-utiliza-novo-larvicida-contra-mosquito

37. Agência Nacional de Vigilância Sanitária - Anvisa. Esclarecimento para o registro de repelentes de insetos. Brasília: Agência Nacional de Vigilância Sanitária; 2018.

38. World Health Organization - WHO. Guidelines for efficacy testing of mosquito repellents for human skin. Geneva: World Health Organization; 2009[acesso 24 jul 2019]. Disponivel em: https://apps.who.int/iris/bitstream/ handle/10665/70072/WHO_HTM_NTD_WHOPES_2009.4_eng. pdf?sequence $=1$

39. Agência Nacional de Vigilância Sanitária - Anvisa. Ouvidoria: protocolo 2019003473. Brasília: Agência Nacional de Vigilância Sanitária; 2019.

40. Hilton L. Zika virus: top mosquito repellent recommendations. Contemp Pediatr. 2016;33(6):14-6.

41. Nguyen QBD, Vu MAN, Hebert AA. Insect repellents: an updated review for the clinician. J Am Acad Dermatol. 2018. https://doi.org/10.1016/j.jaad.2018.10.053

42. US Environmental Protection Agency - EPA. Find the repellent that is right for you. Washington: US Environmental Protection Agency; 2019[acesso 27 out 2019]. Disponível em: https: / www.epa.gov/insect-repellents/ find-repellent-right-you\#search\%20tool
43. US Environmental Protection Agency - EPA. Skin-applied repellent ingredients. Washington: US Environmental Protection Agency; 2019[acesso 27 out 2019]. Disponivel em: https://www.EPA.gov/insect-repellents/ skin-applied-repellent-ingredients

44. Centers for Disease Control and Prevention - CDC. Dengue: prevent mosquito bites. Atlanta: Centers for Disease Control and Prevention; 2019[acesso 6 ago 2019]. Disponível em: https://www.cdc.gov/dengue/prevention/preventmosquito-bites.html

45. NPIC Product Research Online - NPRO. Home. Washington: National Pesticide Information Center; 2019[acesso 30 set 2019]. Disponivel em: http://npic.orst.edu/NPRO/

46. Centers for Disease Control and Prevention - CDC. Surveillance and control of Aedes aegypti and Aedes albopictus in the United States. Atlanta: Centers for Disease Control and Prevention; 2017[acesso 13 set 2019]. Disponível em: https://www.cdc.gov/chikungunya/pdfs/ Surveillance-and-Control-of-Aedes-aegypti-and-Aedesalbopictus-US.pdf

47. European Chemicals Agency - ECHA. Understanding BPR. Helsinki: European Chemicals Agency; 2019[acesso 13 out 2019]. Disponível em: https://echa.europa.eu/regulations/ biocidal-products-regulation/understanding-bpr

48. European Chemicals Agency - ECHA. Information on biocides: biocidal active substances. Helsinki: European Chemicals Agency; 2019[acesso 30 out 2019]. Disponível em: https://echa.europa.eu/information-on-chemicals/ biocidal-active-substances

49. European Centre for Disease Prevention and Control - ECDC. Aedes aegypti: factsheet for experts. Solna: European Centre for Disease Prevention and Control; 2016[acesso 30 out 2019]. Disponível em: https://www.ecdc.europa. eu/en/disease-vectors/facts/mosquito-factsheets/ aedes-aegypti

50. Bragança I, Grosso C, Rede D, Sousa SR, Lemos PC, Domingues VF et al. Ecotoxicological effects of insecticides in plants assessed by germination and other phytotoxicity tools. In: Vats S, editor. Biotic and abiotic stress tolerance in plants. Cham: Springer; 2018. p. 47-76.

51. Santos VSV, Caixeta ES, Campos Júnior EO, Pereira BB. Ecotoxicological effects of larvicide used in the control of Aedes aegypti on nontarget organisms: redefining the use of pyriproxyfen. J Toxicol Environ Health A. 2017;80(3):155-60. https://doi.org/10.1080/15287394.2016.1266721

52. Moura JAS. Avaliação da ecotoxicidade em organismos aquáticos de dois larvicidas para controle do Aedes aegypti: dodecanol (natural) x Piriproxifeno (sintético) [dissertação]. Recife: Universidade Federal de Pernambuco; 2019.

53. Busquet F, Strecker R, Rawlings JM, Belanger SE, Braunbeck T, Carr GJ et al. OECD validation study to assess intra- and inter-laboratory reproducibility of the zebrafish embryo toxicity test for acute aquatic toxicity testing. Regul Toxicol Pharmacol. 2014;69(3):496-511. https://doi.org/10.1016/j.yrtph.2014.05.018 
54. Santos VSV, Campos CF, Campos Júnior EO, Pereira BB. Acute ecotoxicity bioassay using Dendrocephalus brasiliensis: alternative test species for monitoring of contaminants in tropical and subtropical freshwaters. Ecotoxicology. 2018;27(6):635-40. https://doi.org/10.1007/s10646-018-1951-3

55. Carvalho ACC, Quevedo CMG, Machado AA, Machado Neto JG, Paganini WS. Dissipação do malathion da formulação comercial Fyfanon ${ }^{\circledR}$ 96,5\% em águas com diferentes valores de $\mathrm{pH}$. In: Anais do Simpósio Internacional de Microbacias Hidrográficas; Botucatu, Brasil. Botucatu: Universidade de São Paulo; 2017.

56. Moura JAS, Souza-Santos LP. Environmental risk assessment (ERA) of pyriproxyfen in non-target aquatic organisms. Aquat Toxicol. 2020;222. https://doi.org/10.1016/j.aquatox.2020.105448

57. Abe FR, Coleone AC, Machado AA, Machado Neto JG. Ecotoxicity and environmental risk assessment of larvicides used in the control of Aedes aegypti to Daphnia magna (crustacea, cladocera). J Toxicol Environ Health A. 2014;77(1/3):37-45. https://doi.org/10.1080/15287394.2014.865581

58. Abe FR, Machado AA, Coleone AC, Cruz C, Machado Neto JG. Toxicity of diflubenzuron and temephos on freshwater fishes: ecotoxicological assays with Oreochromis niloticus and Hyphessobrycon eques. Water Air Soil Pollut. 2019;230(3). https://doi.org/10.1007/s11270-019-4128-7

59. Marina CF, Bond JG, Muñoz J, Valle J, Novelo-Gutiérrez R, Williams T. Efficacy and non-target impact of spinosad, bti and temephos larvicides for control of Anopheles spp. in an endemic malaria region of southern Mexico. Parasit Vectors. 2014;7(1):1-10. https://doi.org/10.1186/1756-3305-7-55
60. Haya K. Toxicity of pyrethroid insecticides to fish. Environ Toxicol Chem. 1989;8(5):381-91. https://doi.org/10.1002/etc.5620080504

61. Ullah S, Li Z, Zuberi A, Arifeen MZU, Baig MMFA. Biomarkers of pyrethroid toxicity in fish. Environ Chem Lett. 2019;17(2):945-73. https: / /doi.org/10.1007/s10311-018-00852-y

62. Scholz NL, Hopkins WA. Ecotoxicology of anticholinesterase pesticides: data gaps and research challenges. Environ Toxicol Chem. 2006;25(5):1185-6. https://doi.org/10.1897/05-699.1

63. Lawler SP. Environmental safety review of methoprene and bacterially-derived pesticides commonly used for sustained mosquito control. Ecotoxicol Environ Saf. 2017;139:335-43. https://doi.org/10.1016/j.ecoenv.2016.12.038

64. Eurpean Comission - EC. Mosquito pesticide causes knock on problems for birds. News Alert Issue 212. 7 out 2010[acesso 3 out 2019]. Disponível em: https://ec.europa.eu/environment/ integration/research/newsalert/pdf/212na5_en.pdf

65. Aronson D, Weeks J, Meylan B, Guiney PD, Howard PH. Environmental release, environmental concentrations, and ecological risk of $\mathrm{N}, \mathrm{N}$-diethyl-m-toluamide (DEET). Integr Environ Assess Manag. 2012;8(1):135-66. https://doi.org/10.1002/ieam.271

66. Lapied B. Editorial: insecticide mode of action: from insect to mammalian toxicity. Curr Med Chem. 2017;24(27):29101. https://doi.org/10.2174/092986732427170921163124

67. Silva RL, Demarque DP, Dusi RG, Sousa JPB, Albernaz LC, Espindola LS. Residual larvicidal activity of quinones against Aedes aegypti. Molecules. 2020 Sep;25(17):3978. https://doi.org/10.3390/molecules25173978

\section{Agradecimentos}

Os autores agradecem o Projeto ArboControl Brasil, Processo TED n 74/2016 - Acordo de cooperação entre o Ministério da Saúde e a Universidade de Brasília, por fornecer as bolsas de pesquisa.

Contribuição dos Autores

Magalhães NMG - Concepção, planejamento (desenho do estudo), aquisição, análise, interpretação dos dados e redação do trabalho. Silva RL - Planejamento (desenho do estudo), interpretação dos dados e redação do trabalho. Espindola LS - Interpretação dos dados e redação do trabalho. Todos os autores aprovaram a versão final do trabalho. 